\title{
Wavelet-based de-noising of positron emission tomography scans
}

\author{
Wolfgang Stefan, Kewei Chen† Hongbin Guo; Rosemary A. Renaut ${ }^{\S}$ and Svetlana Roudenko
}

August 19, 2011

\begin{abstract}
A method to improve the signal-to-noise-ratio (SNR)of positron emission tomography (PET) scans is presented. A wavelet-based image decomposition technique decomposes an image into two parts, one which primarily contains the desired restored image and the other primarily the remaining unwanted portion of the image. Because the method is based on a texture extraction model that identifies the desired image in the space of bounded variation, these restorations are approximations of piecewise constant images, and are referred to as the cartoon part of the image. Here an approximation using a wavelet decomposition is used which allows solutions to be computed very efficiently. To process 3-D volume data a slice by slice approach in all three directions is adopted. Using a redundant discrete wavelet transform, 3-D restorations can be efficiently computed on standard desktop computers. The method is illustrated for PET images which have been reconstructed from simulated data using the expectation maximization algorithm. When post-processed by the presented wavelet decomposition they show a significant increase in SNR. It is concluded that the new wavelet based method can be used as an alternative to the well established de-noising of PET scans by smoothing with a Gaussian point spread function. In particular, if the volume data are reconstructed using the EM algorithm with a larger number of iterations than the number of iterations that would be used without post-processing, the 3-D images are sharper and show more detail. A MATLAB ${ }^{\circledR}$ based graphical user interface is provided that allows easy exploration of the impact of parameter choices.
\end{abstract}

\section{Introduction and background}

Positron emission tomography (PET) is an image acquisition tool for non-invasively obtaining functional images of an interior organ of interest from a patient [28]. A tiny amount of radioactive tracer is injected into the patient's body and emits gamma ray photons which are collected by multi-ring detector arrays [26]. The physical data, which are photon counts along lines between any two detectors, are contaminated with Poisson noise and stored as a sinogram. The image showing the concentration of the radioactive tracer in the brain is the output of an image reconstruction algorithm such as the expectation maximization (EM) Algorithm, [32, 19, 38, 13]. The underlying process is ill conditioned and therefore yields very noisy and blurred images [32]. Moreover, the noise in the reconstructed image no longer follows a Poisson distribution, and the high noise level makes the quantitative interpretation of the image difficult. This is especially problematic if the image is to be used as input to a pattern classification algorithm, as might be the case for disease detection. Possible applications include tumor detection, especially detection at an early stage when the tumor is small [40], myocardial perfusion deficit [5] and hypometabolism of glucose in studies of early diagnosis of Alzheimer's disease [29, 21, 2, 34]. In such applications it is particularly important to have

\footnotetext{
*Department of Applied and Computational Mathematics, Rice University, wolfgang.stefan@rice.edu

${ }^{\dagger}$ Director, Computational Image Analysis Program, Banner Alzheimer Institute \& and Banner Good Samaritan Medical Center, Kewei.Chen@bannerhealth.com

${ }^{\ddagger}$ School of Mathematical \& Statistical Sciences, Arizona State University, hb_guo@asu.edu

$\S$ School of Mathematical \& Statistical Sciences, Arizona State University, renaut@math.asu.edu

ISchool of Mathematical \& Statistical Sciences, Arizona State University, svetlana@math.asu.edu
} 
images that do not contain much noise and have sharp edges. Images with poor signal to noise ratios, or which are blurred, can lead to mis-diagnosis.

Noise reduction, in particular for Poisson noise, has been addressed by a variety of methods [20, 23]. Traditionally, to reduce noise and artifacts, a de-noising method is applied to the EM reconstruction. The most common method is to convolve the 3D PET reconstruction with a Gaussian point spread function (PSF), which removes noise, but also introduces blur in the image, leading to a loss of fidelity for finding small structures within the image. More recently, a 2-D wavelet thresholding approach which is based on thresholding wavelet coefficients has been proposed, [18,33], but presents only limited flexibility in removing unwanted structures at different scales. Ideally, the de-noising technique should be able to preserve edges in the images. In [31] the authors describe a de-noising method based on $L^{1}$ minimization which is able to preserve edges but is computationally very expensive. Although many advances have been made to decrease the costs in recent years, see e.g. [16, 17, 36, 37, 39], the application to 3D volume data is still too expensive and memory intensive because there is no explicit solution for the minimum $L^{1}$ problem. Instead a numerical optimization has to be performed.

Here a fast method, using a wavelet decomposition based on a model similar to that in [31] is presented. It allows high flexibility in removing structures of different sizes and can be applied to 3D volume images after reconstructing the image from the recorded data using the standard EM algorithm [32, 19]. For examination of the proposed method we could compare with a number of existing approaches. For example, we could contrast with noise level thresholding which also introduces the need to determine appropriate levels of thresholds for all decomposition levels and directions. While such wavelet-thresholding is apparently not yet a widely-adopted approach in the neuroimaging community, noise reduction using smoothing as implemented in SPM is routinely used to increase the signal to noise ratios for neuroimaging data analyses. We thus contrast with the smoothing using SPM, hence providing a useful means to validate the method against a standard accepted by the neuroimaging community. We note also that as compared to wavelet thresholding the proposed method reduces the number of free parameters to only three. The choices for these parameters can easily be explored in an available graphical user interface (GUI) [35]. The GUI enables users to easily load volume data in ANALYSE or NIFTI format and to explore the effect of different choices of the parameters on the solution in real time. Moreover, under standard usage we anticipate that parameters would be determined under standard conditions, for example these would include aspects of the given protocol including imaging time, tracer concentration, and scanner resolution. Thus eliminating the need to estimate appropriate parameter choices for each and every scan. Our major contribution is that we have extended the method introduced in $[12,11]$ for potential practical impact in medical imaging. Originally this method was introduced for application to 2D images and only tested on photographs. Here the method is applied to to de-noise 3D PET data sets and is carefully contrasted with the standard medical approach which is to apply smoothing using the SPM software package. To obtain reproducible results we apply both methods to synthetic data. A careful and more comprehensive evaluation using real data is planned for the future.

The outline of the paper is as follows. Section 2 presents a brief history and introduction of a method proposed for image processing in [31]. It should be noted that this method is not based on statistical estimates but rather uses features of the expected signal, in particular sparseness properties of the gradient, to remove unwanted portions of the image. The numerical implementation of the method and the processing of $3 \mathrm{D}$ volume data is described in Sections 2.1 and 2.2, respectively. A detailed description of the medial imaging restoration problem using simulated data is described in Section 3. It is concluded in Section 4 that this novel approach to de-noising provides an easy way to improve the SNR of reconstructed PET images.

\section{Image decomposition in Besov spaces using wavelets}

Rudin et. al. [31] proposed a decomposition for $2 D$ images $f$ in the form $u+v$ using the assumption that $u$ is modeled in the space of $B V$ functions (functions of bounded variation) and that the texture (noise) $v$ is in the $L^{2}$ space. The space $B V$ was proposed because functions with highly oscillatory contents including noise have a large norm measured in $B V$. Restorations $u$ using this model are piecewise constant and are often referred to as the cartoon part. 
The decomposition is generated by minimizing the convex functional $E(u)=\|u\|_{B V}+\lambda\|f-u\|_{L^{2}}$ over all $u \in B V$. Ideally, $v$ should be found in a space dual to $B V$ but because its dual space is not a function space Meyer [25] proposed using the abstract space $G$, which is a Banach space of functions $f$ such that $f=\triangle g$ with $g \in L^{\infty}$. This approach, however, is not practical and thus other ideas have been suggested including use of the Sobolev space $H^{-1}$ or more generally $H^{-\beta}, \beta \in \mathbb{R},[27,24]$. Most of these methods use a convex optimization approach for minimizing the $B V$ norm. These methods and numerical implementations are very expensive, in part, because $B V$ cannot be characterized by a countable basis. A modification of the space $B V$ to the slightly smaller (inhomogeneous) Besov space $B_{1}^{1,1}$, that can be characterized by wavelet bases, was suggested in [12] for image reconstruction problems. Because a continuous function in $B_{1}^{1,1}$ can be characterized by discrete (wavelet) coefficients a faster algorithm results, of efficiency comparable to that of the fast Fourier transform (FFT), $\left(O\left(n^{2} \log \left(n^{2}\right)\right)\right.$ for a 2D image of $n^{2}$ pixels. Thus, the near optimal components $u$ and $v$ may be found by minimizing

$$
E(u, v)=\|f-(u+v)\|_{L^{2}}^{2}+2 \alpha\|u\|_{B_{1}^{1,1}}+\gamma\|v\|_{H^{-1}}^{2},
$$

where a wavelet characterization for the Besov space $B_{1}^{1,1}$ as well as for $H^{-1}$ can be applied and $\alpha$ and $\gamma$ are two parameters that have to be chosen ${ }^{1}$.

To obtain a representation of (1) we introduce a wavelet decomposition of a function $f$. Let $\left\{\psi_{\lambda}\right\}_{\lambda \in J}$ be a compactly supported wavelet system in $2 \mathrm{D}$, which is generated from any suitably-supported Daubechies wavelets, [10], or their bi-orthogonal correspondents [7] and $\lambda=(i, k, j)$ be a multi index. Then the wavelet decomposition of a function $f$ is

$$
f=\sum_{\lambda \in J}\left\langle f, \tilde{\psi}_{\lambda}\right\rangle \psi_{\lambda} \equiv \sum_{\lambda \in J} f_{\lambda} \tilde{\psi}_{\lambda}
$$

where $\tilde{\psi}_{\lambda}$ forms a bi-orthogonal system and the inner product is chosen appropriately for the Hilbert space that is considered. Its norm in Besov space $B_{p}^{\beta, p}, p \geq 1, \beta \in \mathbb{R}$, is equivalent to

$$
\|f\|_{B_{p}^{\beta, p}} \sim\left(\sum_{\lambda \in J} 2^{|\lambda|\left(\beta+1-\frac{2}{p}\right) p}\left|\left\langle f, \tilde{\psi}_{\lambda}\right\rangle\right|^{p}\right)^{1 / p},
$$

where $|\lambda|$ is the level of the multi level decomposition. Because $L^{2}=B_{2}^{0,2}$ and $H^{-1}=B_{2}^{-1,2}$, the functional in (1) yields the discrete wavelet space representation with wavelet coefficients $\left\{f_{\lambda}\right\},\left\{u_{\lambda}\right\},\left\{v_{\lambda}\right\}$ corresponding to their functions $f, u$ and $v$, respectively,

$$
S(u, v)=\sum_{\lambda \in J}\left(\left|f_{\lambda}-\left(u_{\lambda}+v_{\lambda}\right)\right|^{2}+2 \alpha\left|u_{\lambda}\right|+2^{-2|\lambda|} \gamma\left|v_{\lambda}\right|^{2}\right) .
$$

As shown in [11], $S(u, v)$ is minimized by the closed form minimizer

$$
\begin{aligned}
& \tilde{v}_{\gamma, \alpha}=\sum_{\lambda \in J_{j_{0}}}\left(1+\gamma 2^{-2|\lambda|}\right)^{-1}\left[f_{\lambda}-S_{\alpha\left(2^{2|\lambda|}+\gamma\right) / \gamma}\left(f_{\lambda}\right)\right] \psi_{\lambda} \text { and } \\
& \tilde{u}_{\gamma, \alpha}=\sum_{\lambda \in I_{j_{0}}}\left\langle f, \phi_{j_{0}, k}\right\rangle \phi_{j_{0}, k}+\sum_{\lambda \in J_{j_{0}}} S_{\alpha\left(2^{2|\lambda|}+\gamma\right) / \gamma}\left(f_{\lambda}\right) \psi_{\lambda},
\end{aligned}
$$

where the soft shrinkage operator $S$ is given by

$$
S_{a}(b)=\frac{b}{|b|} \max (|b|-a, 0)
$$

and $J_{j_{0}}$ is the set of all $\lambda$ with $|\lambda|<j_{0}, I_{j_{0}}$ is the the set of all $\lambda$ with $|\lambda|=j_{0}$ and $j_{0}$ is a fixed level, e.g. see [14]. We use $j_{0}=0$ for all numerical computations. Therefore we obtain an approximation of the minimum of (1) by computing the inverse wavelet transform of (4)-(5). For greater flexibility we replace $H^{-1}$ by a class of Sobolev spaces $H^{\beta}$ with $\beta<0$, [30] and [24].

\footnotetext{
${ }^{1}$ Further details on the derivation and usage of the space $B_{1}^{1,1}$ are given in $[6,8,25]$
} 


\subsection{Numerical implementation}

We denote the vector representations of the continuous variables $f, u$ and $v$ by $\mathbf{f}, \mathbf{u}$ and $\mathbf{v}$, respectively. It was demonstrated in [12] that solutions obtained by replacing the continuous wavelet transform in (4)-(5) by the standard decimated discrete Fourier transform (DWT) show the strong presence of artifacts. Moreover, when using the DWT the solution is not translation invariant, which means that a translation of $\mathbf{f}$ does not result in a translation of $\mathbf{u}$ and $\mathbf{v}$. To overcome these problems, cycle spinning of the wavelet basis was proposed in $[12,11]$. Here we propose to use the redundant DWT (RDWT) [3] which omits the step of downsampling and performs instead an upsampling step of the low and high pass filters at each step of the wavelet transform. This procedure is equivalent to keeping two sets of coefficients at each decomposition level, corresponding to keeping either odd or even samples when downsampling the signal. At each level of the reconstruction phase reconstructions obtained by using coefficients from either odd or even downsampling are averaged. The RDWT is translation invariant, i.e., the wavelet transform commutes with the translation operation, see $[9,22,15]$, and is called redundant because it computes more coefficients than the original signal. Hence, the orthogonal discrete wavelet basis in (4)-(5) is replaced by an over-complete wavelet frame. It can be shown, using the RDWT, that there is a weaker norm equivalence than described in (2). Let $f_{\lambda}$ be the image $f$ transformed by the RDWT. Then

$$
A\|f\|^{2} \leq\left\|f_{\lambda}\right\|^{2} \leq B\|f\|^{2} .
$$

It is shown in [15], that $A=2$ and $B=2^{|\lambda|}$ for a $|\lambda|$ scale RDWT operator. The RDWT is also known by a plethora of other names, as listed in [15], including stationary wavelet transform, Algorithme à trous, Quasicontinuous wavelet transform, Translation invariant wavelet transform, Shift invariant wavelet transform, Maximal overlap wavelet transform (MODWT) and Undecimated wavelet transform.

\subsection{Extension to $3 \mathrm{D}$}

The volume data sets used in this study are inherently 3D. If the volumetric data sets were to be processed simply slice by slice, it is likely that a cross section in the two directions perpendicular to the processing direction might show unwanted artifacts. Unfortunately, there is no direct extension of the $2 D$ method described in Section 2. Consequently, a slice by slice approach, along each coordinate axis, for the processing of the $3 D$ images is adopted. Algorithm 1 describes a slice by slice approach in $\mathrm{x}-, \mathrm{y}-$ and $\mathrm{z}$-directions. The three results from each direction are combined by computing their mean value at each voxel.

\section{Algorithm $1 \mathbf{u}+\mathbf{v}$ decomposition of volume data}

1. Given Volume data $\mathbf{f} \in \mathbb{R}^{n_{x} \times n_{y} \times n_{y}}$.

2. For $i=1$ to $n_{x}$ :

3. $\quad$ Process slice by slice in $x$-direction:

4. $\quad \mathbf{f}_{\lambda} \leftarrow \operatorname{RDWT}(\mathbf{f}(i,:,:))$.

5. compute $\mathbf{u}_{\lambda}$ and $\mathbf{v}_{\lambda}$ by (4)-(5), where $2^{-2|\lambda|}$ is replaced by $2^{\beta|\lambda|}$ and $2^{2|\lambda|}$ is replaced by $2^{-\beta|\lambda|}$

6. $\quad \mathbf{u}_{x}(i,:,:) \leftarrow \operatorname{iRDWT}\left(\mathbf{u}_{\lambda}\right)$.

7. $\mathbf{v}_{x}(i,:,:) \leftarrow \operatorname{iRDWT}\left(\mathbf{v}_{\lambda}\right)$.

8. endFor.

9. Process in $y$-direction as in $x$-direction.

10. Process in $z$-direction as in $x$-direction.

11. $\mathbf{u} \leftarrow \frac{1}{3}\left(\mathbf{u}_{x}+\mathbf{u}_{y}+\mathbf{u}_{z}\right)$.

12. $\mathbf{v} \leftarrow \frac{1}{3}\left(\mathbf{v}_{x}+\mathbf{v}_{y}+\mathbf{v}_{z}\right)$.

13. Return $\mathbf{u} \in \mathbb{R}^{n_{x} \times n_{y} \times n_{z}}$ and $\mathbf{v} \in \mathbb{R}^{n_{x} \times n_{y} \times n_{z}}$. 


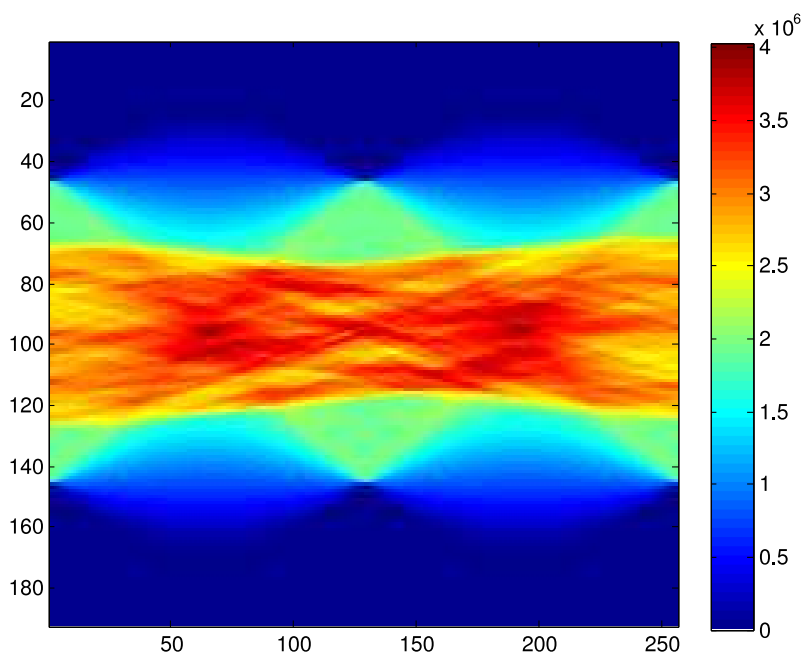

(a) No noise

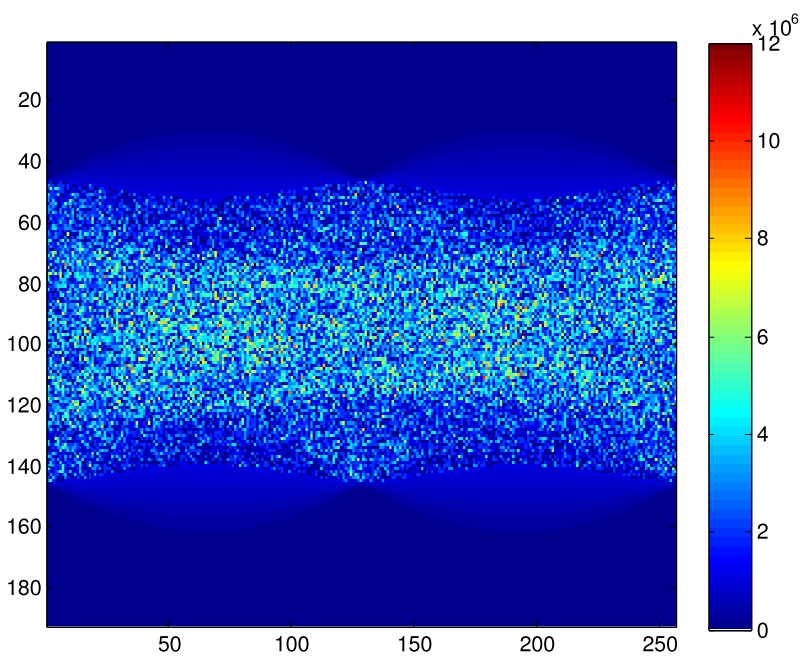

(b) High noise

Figure 1: Sinogram generated from phantom data using (7). Two cases are illustrated (a) the sinogram without noise and (b) the sinogram with high noise contamination $\left(\sigma=5 \cdot 10^{-7}\right)$.

\section{Application in brain PET imaging}

Piecewise constant restorations are desirable in the context of the PET scans, because it is expected that voxel values are similar in similarly activated regions of the brain. We thus evaluate the effectiveness of the new method by applying it to simulated PET data which are generated from the Zubal phantom data. The data is a brain model and can be found in [41]. Each simulated PET slice is generated by computing the sinogram s, adding Poisson noise and reconstructing using the EM algorithm with a fixed number of iterations $N$. The noise-contaminated sinogram is given by

$$
s_{i}=\sigma \mathcal{P}\left(\frac{1}{\sigma}\left(A \mathbf{f}_{\text {true }}\right)_{i}\right),
$$

where $A$ is a $\mathbb{R}^{49152 \times 16384}$ transition matrix and $\mathcal{P}(\cdot)$ is the realization of a Poisson process. Each point in the image domain is projected to a point corresponding to a detector pair of the scanner. As a realization of a Poisson process the noise in the recorded data $s_{i}$ is dependent on the intensity of the signal at each location. To model different noise levels we vary the intensity of the phantom by varying $\sigma$ from $10^{-7}$ to $10^{-4}$. Figure 1 illustrates the recorded data $s_{i}$. In panel (a) the sinogram without noise is shown, i.e., $A \mathbf{f}_{\text {true }}$, and in panel (b) the noise contaminated sinogram using $\sigma=5 \cdot 10^{-7}$ in (7). For examining the algorithm we denote the 3D-image reconstructed using the EM algorithm at iteration $N$ as $\mathbf{f}_{\sigma}^{N}$, and the noise free $N^{\text {th }}$ iteration of the EM algorithm is denoted by $\mathbf{f}_{\infty}^{N}$.

To estimate the parameters for PET data the provided GUI is used. The data is read by pressing the "load SPM volume" and displayed as $2 D$ slices. The parameter values described in the previous paragraph can be used to generate a $2 D$ preview of $\mathbf{u}$ and $\mathbf{v}$ by pressing the "2D preview" button. The parameters are then adjusted until the restoration has the desired noise and level of detail. After appropriate parameters are found the "3D preview" button is pressed to generate the $3 D$ restoration by applying Algorithm 1 to the $3 D$ data volume. The results are saved in ANALYSE format by pressing "save SPM volume".

\subsection{Evaluating the quality of the reconstruction}

The SNR is typically used as a measure of the quality of a given restored signal in relation to a specific reference signal. For a given restored signal $\mathbf{f}$ and reference signal $\hat{\mathbf{f}}$ related by $\hat{\mathbf{f}}=\mathbf{f}+\mathbf{n}$, where $\mathbf{n}$ is 
the noise, the SNR measures the ratio between signal energy and noise energy by $\operatorname{SNR}(\hat{\mathbf{f}})=10 \log _{10} \frac{\hat{\mathbf{f}}^{T} \hat{\mathbf{f}}}{\mathbf{n}^{T} \mathbf{n}}$. Thresholding operations as in (6), however, not only decrease the noise energy but also change the signal energy because the absolute values of the wavelet coefficients are reduced. This may therefore lead to an inaccurate estimate of the SNR. Instead, to achieve a realistic estimate of the SNR the restored signal should be scaled and shifted with respect to the reference image. In this way the noise is given by $\mathbf{n}=\nu \hat{\mathbf{f}}+\tau-\mathbf{f}$, where variables $\nu$ and $\tau$ are the scaling and shifting variables needed to eliminate the effects of scaling and shifting $\mathbf{f}$. Chosing their values so as to maximize the SNR yields the modified SNR computed by

$$
\operatorname{SNR}_{\hat{\mathbf{f}}}(\mathbf{f})=10 \log _{10} \frac{\mathbf{f}^{T} \mathbf{f}}{\left(\nu^{*} \hat{\mathbf{f}}+\tau^{*}-\mathbf{f}\right)^{T}\left(\nu^{*} \hat{\mathbf{f}}+\tau^{*}-\mathbf{f}\right)},
$$

where $\nu^{*}$ and $\tau^{*}$ are the maximizers of (8) and are explicitly given by

$$
\tau^{*}=\frac{1^{T} \mathbf{f}-\left(\hat{\mathbf{f}}^{T} \mathbf{f}\right)\left(1^{T} \hat{\mathbf{f}}\right)}{1-N}, \quad \nu^{*}=\frac{\mathbf{f}^{T} \hat{\mathbf{f}}-1^{T} \hat{\mathbf{f}} \tau^{*}}{\hat{\mathbf{f}}^{T} \hat{\mathbf{f}}} .
$$

The vectors $\mathbf{f}$ and $\hat{\mathbf{f}}$ are assumed to be column vectors in $\mathbb{R}^{n_{x} \cdot n_{y} \cdot n_{z}}$. Note that if an unscaled $\hat{\mathbf{f}}$, i.e. $\nu=1$ and $\tau=0$, is used in (8), the true SNR is under estimated.

Higher SNR values indicate a closer fit of the image $\mathbf{f}$ to the reference image $\hat{\mathbf{f}}$. Figure 2 (a) shows 3 slices of the brain phantom generated using the Zubal phantom $\mathbf{f}_{\text {true }}$. Restorations of these phantom images using $\sigma=10^{-4}$ after 20 and 100 iterations, and $\sigma=10^{-6}$ after 20 iterations are shown in Figures 2 (b)-(d). The SNRs are evaluated using the true phantom image $\mathbf{f}_{\text {true }}$ and the noise free but blurred reconstruction $\mathbf{f}_{\infty}^{N}$.

After 20 iterations the reconstructions contain only a moderate amount of noise as compared to those obtained after 100 iterations $\left(\operatorname{SNR}_{\mathbf{f}_{\infty}^{20}}\left(\mathbf{f}_{10^{-4}}^{20}\right)>\operatorname{SNR}_{\mathbf{f}_{\infty}^{100}}\left(\mathbf{f}_{10^{-4}}^{100}\right)\right)$. However, $\mathbf{f}_{10^{-4}}^{100}$ is less corrupted by blurring than is $\mathbf{f}_{10^{-4}}^{20}$. This experiment suggests that, at least up to some unknown maximal number of iterations, the errors in the reconstructions are of two types: i) a truncation error in the form of blurring, because of a small number of iterations and ii) error due to the noise amplification caused by the ill-posedness of the reconstruction problem. In the following we introduce the notation $\mathbf{f}_{1}:=\mathbf{f}_{10^{-4}}^{100}$ and $\mathbf{f}_{2}:=\mathbf{f}_{10^{-6}}^{20}$.

\subsection{Restoration by the $\mathrm{u}+\mathrm{v}$ decomposition}

We restore the phantom data by applying Algorithm 1 to reconstructions $\mathbf{f}_{1}$ and $\mathbf{f}_{2}$ using fixed parameters $\gamma=5 \cdot 10^{-3}, \beta=-0.1$ and variable choices for $\alpha$. Bayesian approaches have been used in the past to estimate optimal thresholds for each decomposition level and direction [20,4]. Here we investigate a method that uses a minimal number of parameters, while allowing investigation of the effectiveness of the de-noising method on PET data without using any parameter selection paradigm. It is argued in $[12,11]$ that the ratio $\gamma / \alpha$ determines how much of the texture is moved from $\mathbf{u}$ to $\mathbf{v}$, and the sizes of $\gamma$ and $\alpha$ determine the magnitude of the residual $\mathbf{r}:=\mathbf{f}-\mathbf{u}-\mathbf{v}$. We therefore choose $\gamma$ such that the residual $\mathbf{r}$ can be neglected and only vary $\alpha$. The choice $\beta=-0.1$ permits a large amount of the noise and only a small amount of the brain structure to be moved to $\mathbf{v}$.

It is intended that the $\mathbf{u}+\mathbf{v}$ decomposition be employed as a post-processing SNR improvement step to the EM reconstruction of the PET image. Improvement in SNR is achieved by both reducing the noise in the signal and by increasing the level of detail in the reconstruction. Typically, the EM algorithm uses a relatively small number of iterations so that the noise contamination is limited. Compare, for example, the SNR of Figures 2(a) and (b). There are more details in Figure 2(b) but the noise contamination is also greater. After the $\mathbf{u}+\mathbf{v}$ decomposition the $\mathbf{u}$ part contains mostly the desired signal and most of the noise is moved into $\mathbf{v}$. Thus we expect that $\mathbf{u}$ is closer to the true solution and that the denominator in (8) is small which generates a large SNR.

Figure 5 illustrates the SNR for the low noise case in Figure 2(b). The color shades represent $\operatorname{SNR}_{\mathbf{f}_{\infty}^{N}}(\mathbf{u})$, with the $x$-axis being different choices of $\alpha$ and the $y$-axis the number of iterations of the EM algorithm. The contour lines represent $\operatorname{SNR}_{\mathbf{f}_{\text {true }}}(\mathbf{u})$. Small values of $\alpha$ (left side of the plot) correspond to less de-noising, 

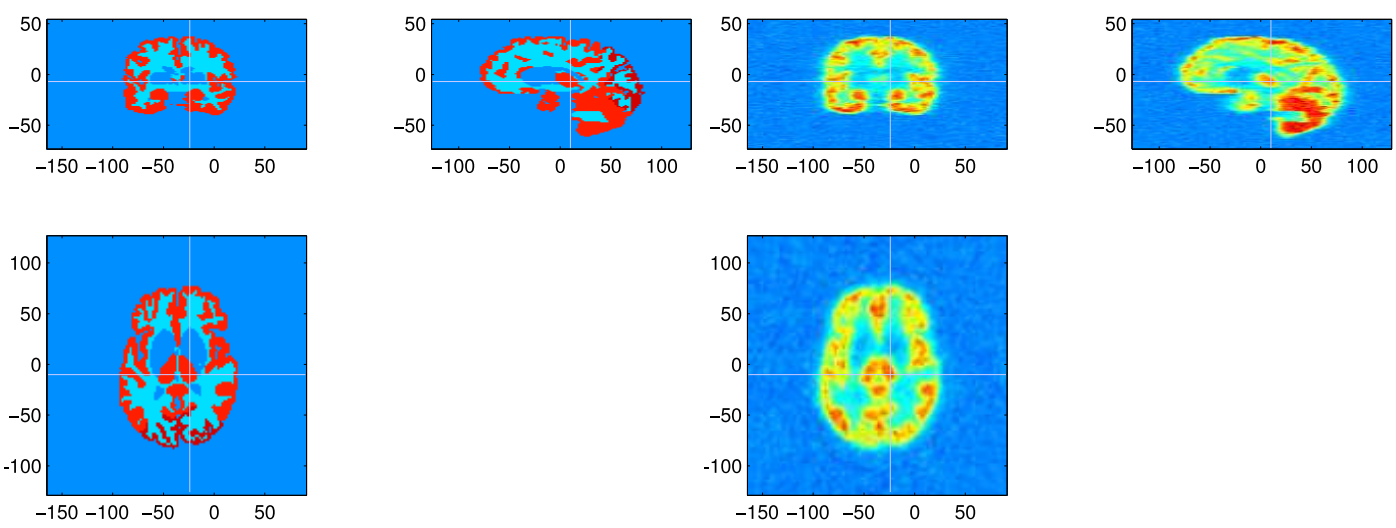

(a) Phantom data $\mathbf{f}_{\text {true }}$
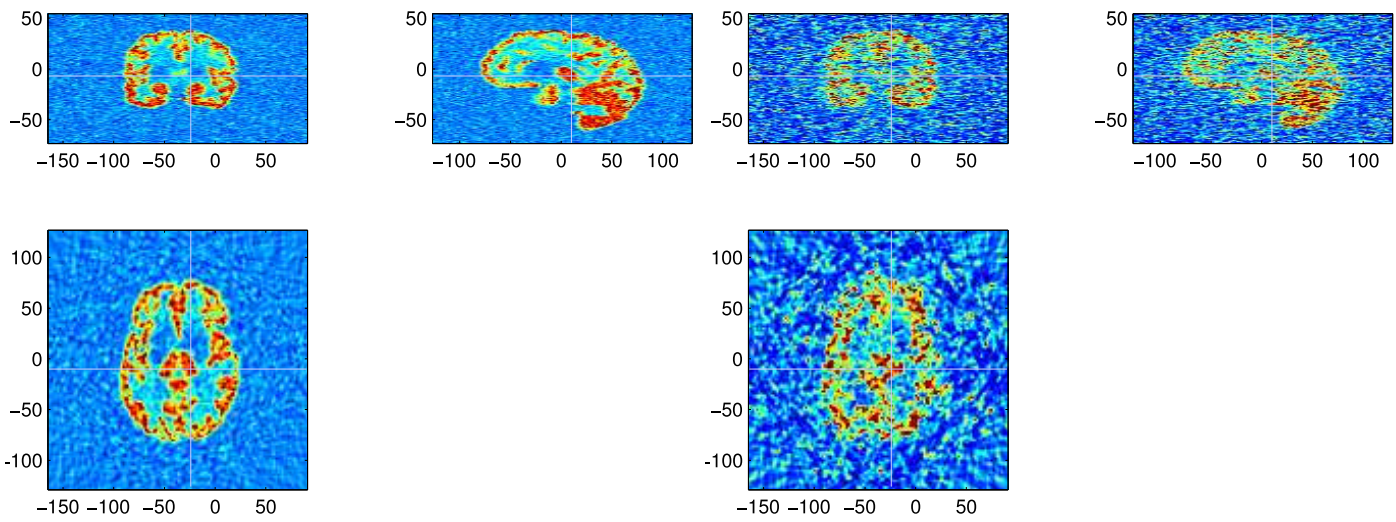

(c) Reconstructed data $\mathbf{f}_{10^{-6}}^{100}$

(d) Reconstructed data $\mathbf{f}_{2}:=\mathbf{f}_{10^{-6}}^{20}$

Figure 2: This figure illustrates the trade-off in the EM algorithm between few iterations and low noise contamination as compared to a larger number of iterations and an image with more details. No postprocessing has been applied. Panel (a) shows images generated from the Zubal phantom without adding blurring or noise. The intensity values are assigned to different brain regions according an approximate ratios gray matter : white matter : CSF of about 2:1:0. Reconstructions using the EM algorithm at various noise levels, generated by varying the intensity of the voxels of the phantom data, are shown. In (b) the case for low noise level: $\sigma=10^{-4}$ after 20 iterations of the EM algorithm $\mathbf{f}_{10^{-4}}^{20}$ with $\operatorname{SNR}_{\mathbf{f}_{\text {true }}}\left(\mathbf{f}_{10^{-4}}^{20}\right)=14.0 d B$ and $\operatorname{SNR}_{\mathbf{f}_{\infty}^{20}}\left(\mathbf{f}_{10^{-4}}^{20}\right)=26.5 \mathrm{~dB}$. In (c) the low noise level case after 100 iterations $\mathbf{f}_{10^{-4}}^{100}$ for which $\mathrm{SNR}_{\mathbf{f}_{\text {true }}}\left(\mathbf{f}_{10^{-4}}^{100}\right)=$ $13.8 d B$ and $\mathrm{SNR}_{\mathbf{f}_{\infty}^{100}}\left(\mathbf{f}_{10^{-4}}^{100}\right)=16.5 d B$. In (d) the high noise level case: $\sigma=10^{-6}$ after 20 iterations of the EM algorithm $\mathbf{f}_{10^{-6}}^{20^{\infty}}$ for which $\operatorname{SNR}_{\mathbf{f}_{\text {true }}}\left(\mathbf{f}_{10^{-6}}^{20}\right)=8.7 d B$ and $\operatorname{SNR}_{\mathbf{f}_{\infty}^{12}}\left(\mathbf{f}_{10^{-6}}^{20}\right)=10.8 d B$. 
larger values to more de-noising. The plot illustrates a basic trade-off property of the EM algorithm: For an increasing number of iterations (left of the plot, from top to bottom) $\operatorname{SNR}_{\mathbf{f}_{\infty}^{N}}(\mathbf{u})$ is decreasing due to the increasing noise in reconstructions with increasing iterations, $\operatorname{SNR}_{\mathbf{f}_{\text {true }}}(\mathbf{u})$ is increasing first, because the reconstructions become sharper with more iterations but start to decrease after about 50 iterations as the noise begins to dominate the overall error. The plot shows that the $\mathbf{u}+\mathbf{v}$ decomposition can be used to increase $\operatorname{SNR}_{\mathbf{f}_{\text {true }}}(\mathbf{u})$, in particular, when more iterations are used. The $\mathbf{u}+\mathbf{v}$ decomposition can therefore be used to delay the onset of the high noise contamination when the EM algorithm is used for reconstruction.

Figure 3 illustrates the advantages of the wavelet method over traditional smoothing using a Gaussian PSF with half-width $8 \mathrm{~mm}$ using the SPM software [1]. Shown in this figure are the true image (a), the noisy reconstructions $\mathbf{f}_{1}$ (b) and $\mathbf{f}_{2}$ (c) (from Figure 2), post-processing of $\mathbf{f}_{1}$ and $\mathbf{f}_{2}$ using SPM, (d) and (g) respectively, and post-processing using the $\mathbf{u}+\mathbf{v}$ decomposition, (e)-(f) and (h)-(i), respectively. The $\mathbf{u}+\mathbf{v}$ decomposition in panels (e)-(f) uses the parameters $\gamma=5 \cdot 10^{-3}, \beta=-0.1$ and $\alpha=0.0115$. Thus $\left\|\mathbf{u}_{1}+\mathbf{v}_{1}-\mathbf{f}_{1}\right\|_{2} /\left\|\mathbf{f}_{1}\right\|_{2}=10^{-4}$, and $\mathbf{f}_{1} \approx \mathbf{u}_{1}+\mathbf{v}_{1}$. For $(\mathrm{e}) \operatorname{SNR}_{\mathbf{f}_{\text {true }}}\left(\mathbf{u}_{1}\right)=16.2 d B$, which is an improvement of $2.3 d B$ as compared to $\mathrm{SNR}_{\mathbf{f}_{\text {true }}}\left(\mathbf{f}_{1}\right)$. Panel (f) shows the content that has been removed from $\mathbf{f}_{1}$; it contains mostly noise and no edge information. Thus the restoration $\mathbf{u}_{1}$ is much sharper than if $\mathbf{f}_{1}$ is smoothed by SPM to remove the noise, see panel (d). For the case with higher noise, $\mathbf{f}_{2}$, the results illustrated in panels (h)-(i) are obtained using the parameters $\gamma=5 \cdot 10^{-3}, \beta=-0.1$ and $\alpha=0.061$. While (i) shows that some portion of the brain outline and brain structure are still evident, indicating that some of the information has been lost from (c), the restored image given in (h) is still sharper than the image obtained by smoothing with $\mathrm{SPM}$, illustrated in (g). In general, post-processing the images using the $\mathbf{u}+\mathbf{v}$ decomposition yields sharper and less noisy images than using smoothing with the standards used in SPM. Figure 4 shows a comparison of different 1D slices of the images in Figure 3 to further illustrate the resolution gain of the proposed method. The 1D slices indicate that the restorations using the wavelet method are sharper than those taken from either the SPM-smoothed image, or from the original EM reconstruction without post-processing but with a comparable noise level.

A MATLAB ${ }^{\circledR}$ based GUI, shown in Figure 6, can be downloaded [35]. It allows the user to load SPM ANALYSE volumes and explore different parameter choices. The implementation is fast enough to see the effects of changes of the parameters in real time.

\section{Conclusions}

The reconstruction of 3D volumes from data collected by a PET scanner using the EM algorithm has been considered. Usually the number of iterations of the EM algorithm is kept low in order to limit the amplification of noise in the data. A post-processing step using the $\mathbf{u}+\mathbf{v}$ decomposition can significantly increase the SNR of restorations. The method is based on the RDWT and can be computed very efficiently using FFTs. Unlike methods that automatically estimate many thresholding parameters using Bayesian estimators, the proposed method uses a user-guided approach to pick appropriate reconstruction parameters. A MATLAB ${ }^{\mathrm{R}}$ GUI that permits easy exploration of the impact of parameter choices of the $\mathbf{u}+\mathbf{v}$ decomposition for SPM volumes in ANALYSE format is available [35]. The code is implemented using the MATLAB ${ }^{\circledR}$ native distributed engine and can run on single CPU desktop computers as well as multi CPU clusters. The $\mathbf{u}+\mathbf{v}$ decomposition of two dimensional slices can be computed in real time and be used to select appropriate parameters. In the current MATLAB ${ }^{\circledR}$ implementation a full $3 \mathrm{D}$ decomposition of a $128 \times 128 \times 128$ scan can be performed on a desktop computer in about 2-3 minutes and about 20s on a computer cluster system with 20 nodes.

\section{Acknowledgement}

Wolfgang Stefan was supported in part by the Arizona Department of Health Services Alzheimer's Research Grant, and NSF grants DMS 0513214 and DMS 0652833. Rosemary Renaut was supported in part by NSF grants DMS 0513214 and DMS 0652833, the Arizona Department of Health Services Alzheimer's Research 
Grant, and by NIH grant EB 2553301. Kewei Chen was in part supported by the Arizona Department of Health Services Alzheimer's Research Grant, and by NIH grant EB 2553301. Svetlana Roudenko was supported in part by NSF grant DMS 0652833 and DMS 0633033. Hongbin Guo was supported by DMS 0513214, Alzheimer's Research Grant, and NIA sponsored Arizona AD Center Pilot study grant.

\section{References}

[1] Statistical Parametric Mapping, Department of Imaging Neuroscience, University College London, http://www.fil.ion.ucl.ac.uk/spm/.

[2] G. E. Alexander, K. Chen, P. Pietrini, S. I. Rapoport, and E. M. Reiman. Longitudinal evaluation of cerebral metabolic decline in dementia: Implications for using resting PET to measure outcome in long-term treatment studies of Alzheimer's disease. American Journal of Psychiatry, 159:738-745, 2002.

[3] C. S. Burrus, R. A. Gopinath, and H. Guo. Introduction to wavelets and wavelet transforms : a primer. Upper Saddle River, N.J. : Prentice Hall, 1998.

[4] S. G. Chang, B. Yu, and M. Vetterli. Adaptive wavelet thresholding for image denoising and compression. IEEE Transactions on Image Processing, 9(9):1532-46, 2000.

[5] K. Chen, M. Lawson, E. M. Reiman, D. Feng, S.-C. Huang, D. Bandy, D. Ho, L.-S. Yun, and A. Palant. Generalized linear least square method for fast generation of myocardial blood flow parametric images with N-13 ammonia PET. IEEE Transactions On Medical Imaging, 17(2):236-243, 1998.

[6] A. Cohen, W. Dahmen, I. Daubechies, and R. DeVore. Harmonic Analysis of the Space BV. Matematica Iberoamericana, 19:235-263, 2003.

[7] A. Cohen, I. Daubechies, and J.-C. Feauveau. Biorthogonal bases of compactly supported wavelets. Communications on Pure and Applied Mathematics, 45(5):485-560, 1992.

[8] A. Cohen, R. DeVore, P. Petrushev, and H. Xu. Nonlinear Approximation and the Space $B V\left(\mathcal{R}^{\in}\right)$. American Journal of Mathematics, 121:587-628, 1999.

[9] J. M. Combes, A. Grossmann, and P. Tchamitchian, editors. Wavelets : Time-frequency methods and phase space : Proceedings of the international conference, Marseille, France, December 14-18, $198 \%$. Springer-Verlag, Berlin ; New York, December 1989.

[10] I. Daubechies. Ten Lectures on Wavelets. SIAM, Philadelphia, PA, 3600 University City Science Center, Philadelphia, Pennsylvania, 1992.

[11] I. Daubechies and G. Teschke. Wavelet based image decomposition by variational functionals. In T. Frédéric, editor, Wavelet Applications in Industrial Processing, volume 5266 of Proceedings of the SPIE, pages 94-105. SPIE, February 2004.

[12] I. Daubechies and G. Teschke. Variational image restoration by means of wavelets: simultaneous decomposition, deblurring, and denoising. Applied and Computational Harmonic Analysis, 19(1), 2005.

[13] A. P. Dempster, N. M. Laird, and D. B. Rubin. Maximum likelihood from incomplete data via the EM algorithm. J. Roy. Stat. Soc., Ser. B, 39:1-38, 1977.

[14] D. L. Donoho and I. M. Johnstone. Ideal spatial adaptation by wavelet shrinkage. Biometrika, 1994.

[15] J. E. Fowler. The redundant discrete wavelet transform and additive noise. IEEE Signal Processing Letters, 12(9), September 2005.

[16] M. Grant, S. Boyd, and Y. Ye. Disciplined Convex Programming, pages 155-210. Nonconvex Optimization and its Applications. Springer, 2006. 


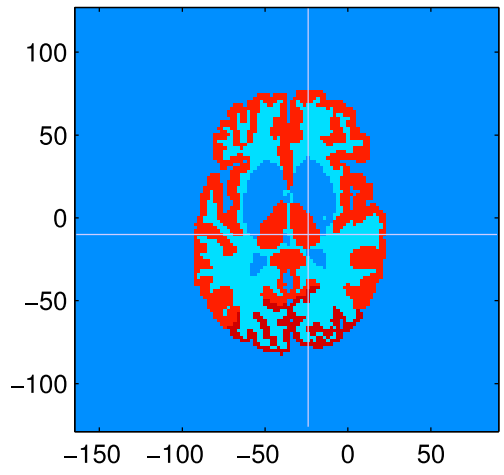

(a) Phantom

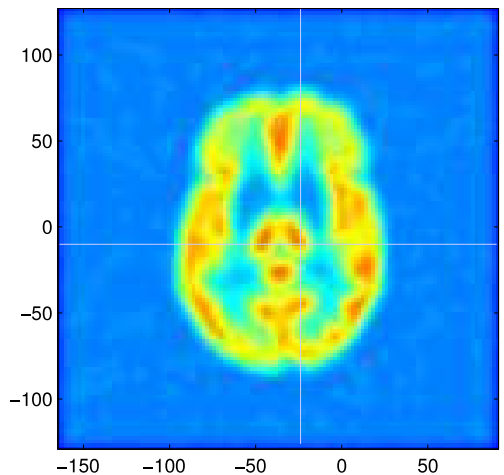

(d) $\mathbf{f}_{1} 8 \mathrm{~mm}$ smoothing

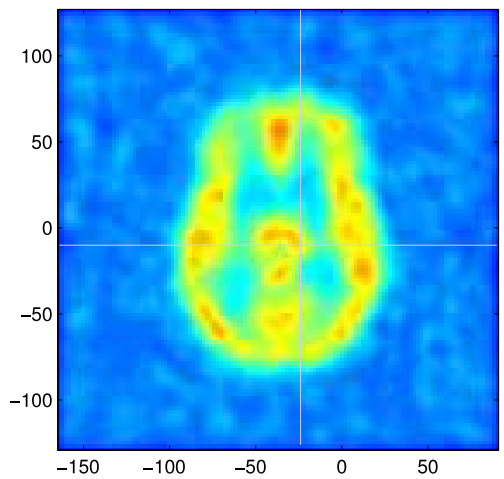

(g) $\mathbf{f}_{2} 8 \mathrm{~mm}$ smoothing

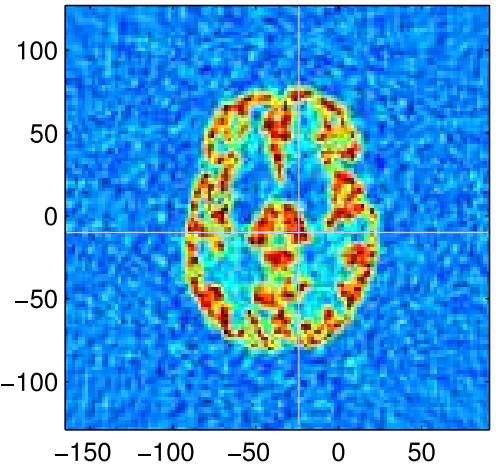

(b) $\mathbf{f}_{1}$

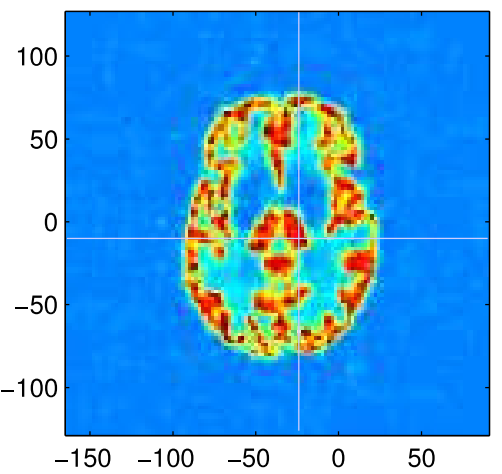

(e) $\mathbf{u}_{1}$

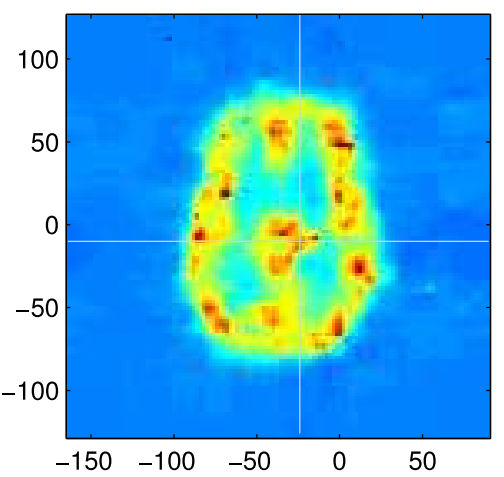

(h) $\mathbf{u}_{2}$

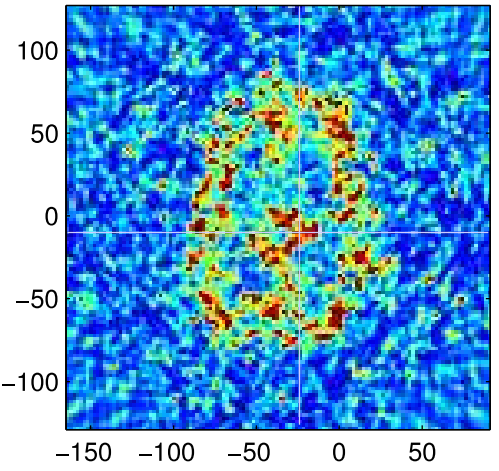

(c) $\mathbf{f}_{2}$

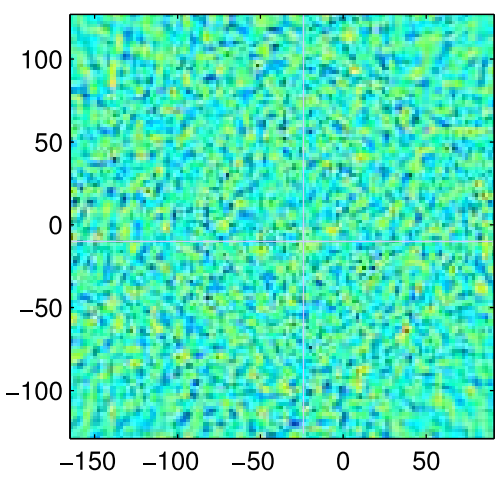

(f) $\mathbf{v}_{1}$

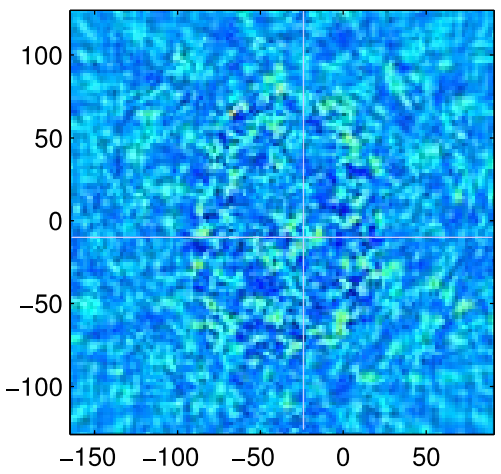

(i) $\mathbf{v}_{2}$

Figure 3: This figure illustrates the impact of post-processing of images $\mathbf{f}_{1}$ and $\mathbf{f}_{2}$ using the wavelet decomposition as compared to using SPM. Panels (a)-(c) are the original phantom, the simulated PET scan already illustrated in Figure 2 (b) but with more detail, and the simulation with more noise, $\mathbf{f}_{2}$, respectively. Panels (d)-(f) illustrate post-processing of the image in (b) using conventional smoothing with SPM and the wavelet decomposition, while panels (g)-(i) show the corresponding results for panel (c). 


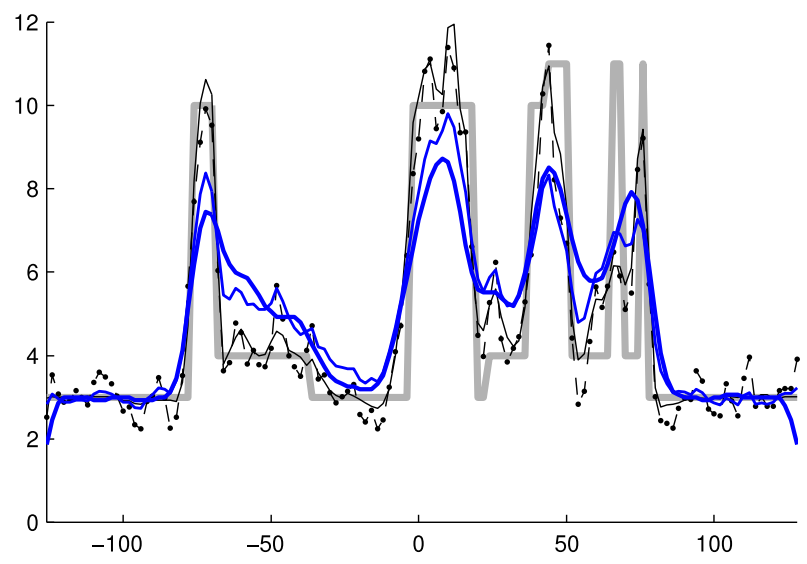

(a) low noise

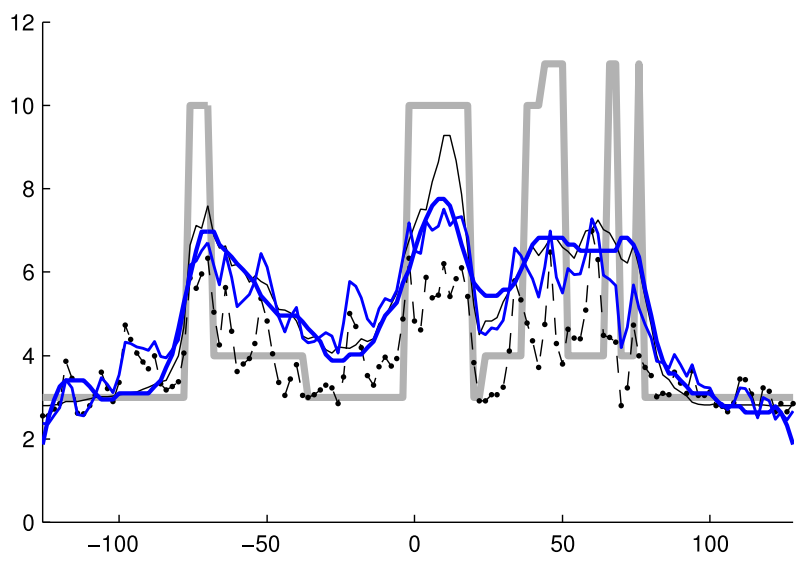

(b) high noise

Figure 4: One dimensional vertical slices of the images in Figure 3. Panel (a) shows the low noise case corresponding to $\mathbf{f}_{1}$ and panel (b) shows the high noise case corresponding to $\mathbf{f}_{2}$. In each plot the thick gray line shows the phantom data, the dashed line with the point markers shows the noisy signals $\mathbf{f}_{1}$ and $\mathbf{f}_{2}$ respectively, the thick blue line (gray in b/w) shows the $8 \mathrm{~mm}$ SPM-smoothed solution, the thin black line shows $\mathbf{u}_{1}$ and $\mathbf{u}_{2}$ respectively and the thin blue (gray in $\mathrm{b} / \mathrm{w}$ ) line shows the EM reconstruction truncated such that the noise level in the signal is comparable to $\mathbf{u}_{1}$ and $\mathbf{u}_{2}$, respectively.

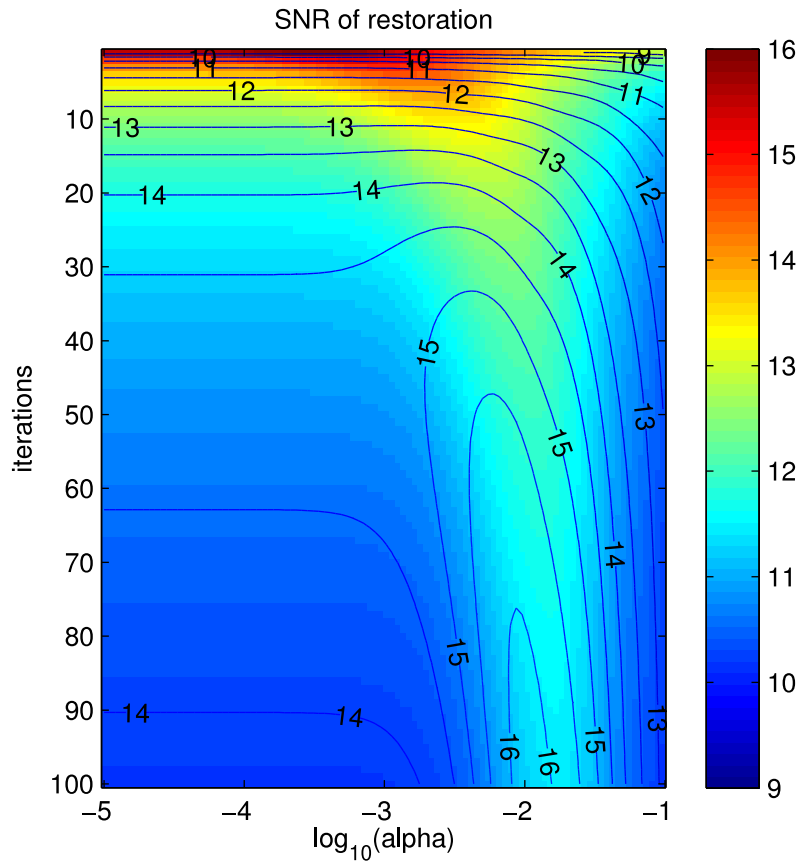

(a) low noise case

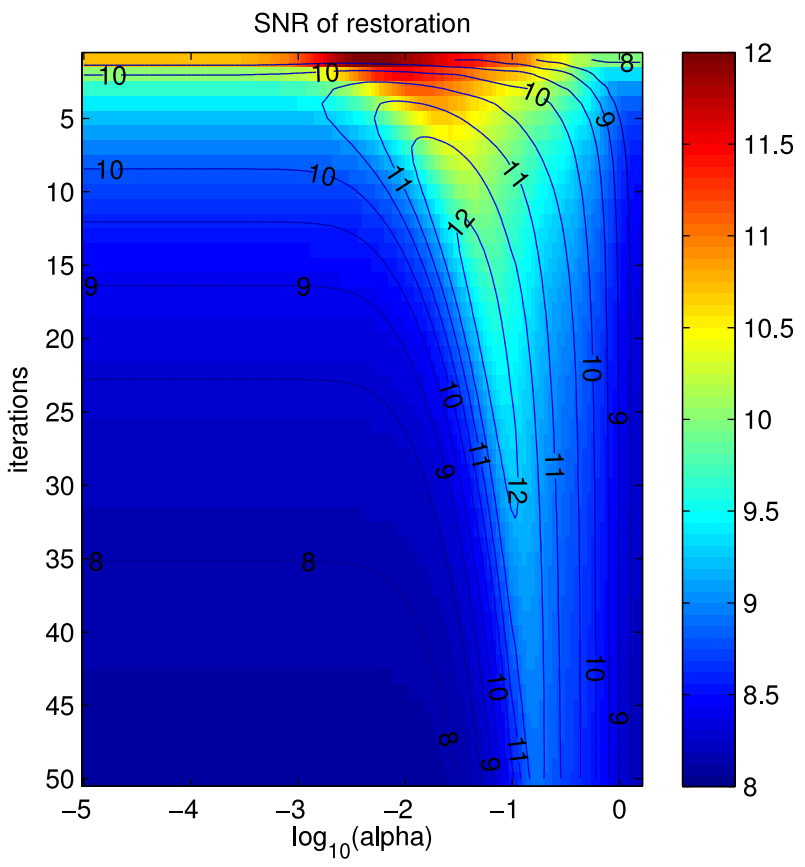

(b) high noise case

Figure 5: SNR measurements of wavelet restoration. The color plot shows SNR measurements $\operatorname{SNR}_{\mathbf{f}_{\infty}^{N}}(\mathbf{u})$, the contour lines show $\operatorname{SNR}_{\mathbf{f}_{\text {true }}}(\mathbf{u})$. The x-axis shows the parameters $\alpha$ and the y-axis the number of iterations of the EM algorithm. 


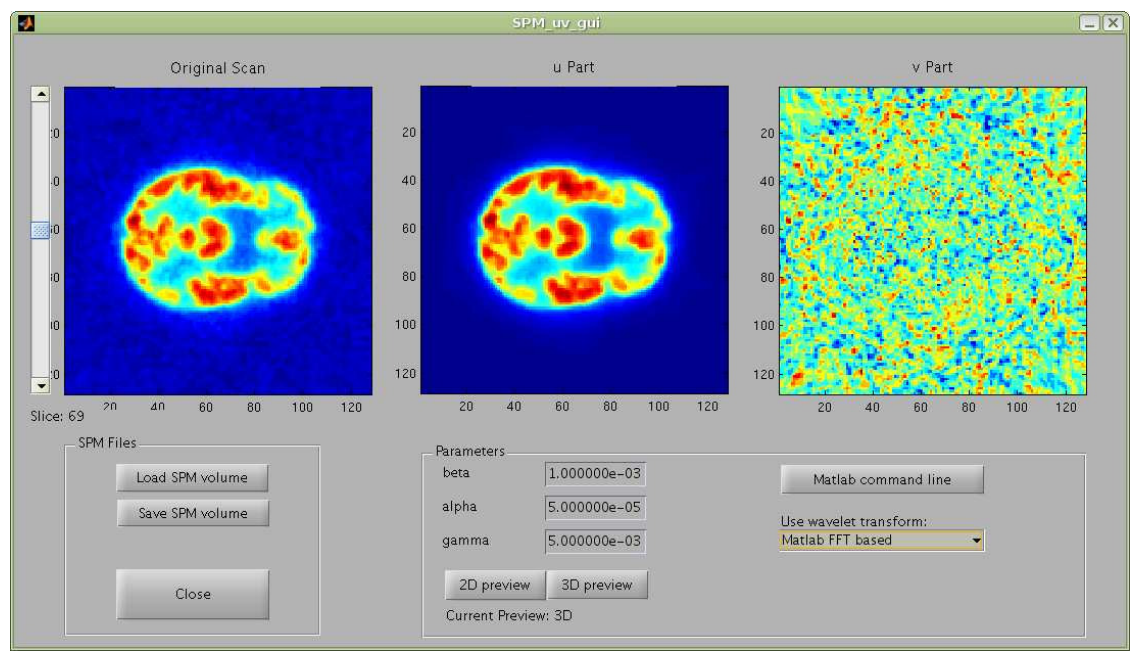

Figure 6: The GUI can be used to read volumes in the SPM ANALYSE format and perform the $\mathbf{u}+\mathbf{v}$ decomposition with different parameters.

[17] M. Grant, S. Boyd, and Y. Ye. CVX: Matlab Software for Disciplined Convex Programming, September 2007.

[18] J.-W. Lin and A. F. Laine and S. R. Bergmann. Improving PET-based physiological quantification through methods of wavelet denoising. IEEE Transactions on Biomedical Engineering, 48(2):202-212, February 2001.

[19] L. Kaufman. Implementing and Accelerating the EM Algorithm for Positron Emission Tomography. IEEE Transactions on Medical Imaging, M1-6(6):37-51, 1987.

[20] N. Khlifa, N. Gribaa, I. Mbazaa, and K. Hamruoni. A based Bayesian wavelet thresholding method to enhance nuclear imaging. International Journal of Biomedical Imaging, 2009:506120, 2009.

[21] K. S. Kwan, A. C. Evans, and G. B. Pike. MRI simulation-based evaluation of image-processing and classification methods. IEEE Transactions on Medical Imaging, 18(11):1085-1097, November 1999.

[22] M. Lang, H. Guo, J. E. Odegard, C. S. Burrus, and R. O. Wells Jr. Noise reduction using an undecimated discrete wavelet transform,. IEEE Signal Processing Letters, 3(1):10-12, 1996.

[23] S. Lefkimmiatis, P. Maragos, and G. Papandreou. Bayesian inference on multiscale models for Poisson intensity estimation: Applications to photon-limited image denoising. IEEE Transactions On Image Processing, 18(8):1724-1741, August 2009.

[24] L. Lieu and L. Vese. Image restoration and decomposition via bounded total variation and negative Hilbert-Sobolev spaces. Applied Math $\& 3$ Optimization, 58(2):167-193, May 2008.

[25] Y. Meyer. Oscillating Patterns in Image Processing and Nonlinear Evolution Equations. University Lecture Series Volume 22. AMS, 2002.

[26] NRC. Mathematics and Physics of Emerging Biomedical Imaging. National Research Council, Institute of Medicine, National Academy Press, Washington DC, 1996. http://www.nas.edu/.

[27] S. Osher, A. Sole, and L. Vese. Image decomposition and restoration using total variation minimization and the $H^{1}$ norm. Multiscale Modeling ES Simulation, 1(3):349-370, 2003. 
[28] M. E. Phelps, J. Mazziotta, and H. Schelbert. Positron Emission Tomorgraphy And Autoradiography, Principles and Applications for the Brain and Heart. Raven Pr, January 1986.

[29] E. M. Reiman, R. J. Caselli, L.-S. Yun, K. Chen, D. Bandy, S. Minoshima, S. Thibodeau, and D. Osborne. Preclinical evidence of a genetic risk factor for Alzhemer's disease in apolipoprotein E type 4 homozygotes using Positron Emission Tomography. The New England Journal of Medicine, 334:752-758, 1996.

[30] S. Roudenko. Noise and texture detection in image processing. LANL report: W-7405-ENG-36, 2004.

[31] L. I. Rudin, S. Osher, and E. Fatemi. Nonlinear total variation based noise removal algorithms. Physica $D, 60: 259-268,1992$.

[32] L.A. Shepp and Y. Vardi. Maximum likelihood reconstruction for emission tomography. IEEE Transactions on Medical Imaging, MI-1(2):113-122, 1982.

[33] Y. Y. Shih, J. C. Chen, and R. S. Liu. Development of wavelet de-noising technique for PET images. Computerized Medical Imaging and Graphics, 29(4):297-304, April 2005.

[34] D. H. Silverman, G. W. Small, C. Y. Chang, C. S. Lu, D. Kung, M. A. Aburto, W. Chen, J. Czernin, S. I. Rapoport, P Pietrini, G. E. Alexander, M. B. Schapiro, W. J. Jagust, J. M. Hoffman, K. A. Welsh-Bohmer, A. Alavi, C. M. Clark, E. Salmon, M. J. de Leon, R. Mielke, J. L. Cummings, A. P. Kowell, S. S. Gambhir, C. K. Hoh, M. E. Phelps, et al. Positron emission tomography in evaluation of dementia: Regional brain metabolism and long-term outcome. JAMA : the Journal of the American Medical Association, 286(17):2120-2127, 2001.

[35] W. Stefan, S. Roudenko, K. Chen, R. A. Renaut, and H. Guo. Software for Denoising of 3D SPM Analyse Volumes. Arizona State University, August 2008. http://mathpost.la.asu.edu/ stefan/ spm_uv_gui.html.

[36] J. F. Sturm. Using SeDuMi 1.02, a MATLAB toolbox for optimization over symmetric cones. $O p$ timization Methods and Software, 11-12:625-653, 1999. Special issue on Interior Point Methods (CD supplement with software), http://sedumi.mcmaster.ca/.

[37] K. Toh, R. Tütüncü, and M. Todd. SDPT3 4.0 (beta) (software package). Technical report, Department of Mathematics National University of Singapore, September 2006. http://www.math.nus.edu.sg/ $\sim$ mattohkc/sdpt3.html.

[38] Y. Vardi, L. A. Shepp, and L. Kaufman. A statistical model for positron emission tomogrphy. Journal of the American Statistical Association, 80:8-20, Mar. 1985.

[39] Y. Wang, W. Yin, and Y. Zhang. A fast algorithm for image deblurring with total variation regularization. SIAM Journal on Imaging Sciences, 1(3):248-272, 2008.

[40] C. Zhao, Z. Chen, X. Ye, Y. Zhang, T. Aburano, M. Tian, and H. Zhang. Imaging a pancreatic carcinoma xenograft model with 11C-acetate: a comparison study with 18F-FDG. Nucl Med Commun, Epub ahead of print, August 2009.

[41] I. G. Zubal, C. R. Harrell, Z. Rattner, G. Gindi, and P. B. Hoffer. Computerized three-dimensional segmented human anatomy. Medical Physics, 21(2):299-302, 1994. 